\title{
Some General Results Concerning the Critical Exponents of Percolation Processes
}

\author{
Richard Durrett * \\ Dept. of Mathematics, University of California, Los Angeles, California 90024, USA
}

\begin{abstract}
Summary. In this paper we will give some results concerning the critical exponents of percolation processes which are valid for "any" model. These results show that in several respects the behavior which occurs for percolation on the binary tree provides bounds on one side for what happens in general. These results and their proofs are closely related to their analogues for the Ising model.
\end{abstract}

\section{Introduction, Description of Models}

In order to state the results which have been obtained for the critical exponents of percolation processes we have to first describe the class of models to which our results can be applied. To use a little legal jargon, this includes but is not limited to the following four examples:

1. Bond Percolation in $Z^{d}$. We consider $Z^{d}$ as a graph with edges connecting all points $x$ and $y$ which are "nearest neighbors" (i.e. $\|x-y\|_{1}=1$ ). Each edge is independently open with probability $p$ and closed with probability $1-p$. We think of open bonds as permitting us to go along the bond and with this in mind we make the following definitions

$$
x \rightarrow y \quad(y \text { can be reached from } x \text { or } x \text { leads to } y)
$$

if there is an open path from $x$ to $y$ : that is, there is a sequence $x_{0}$ $=x, x_{1}, \ldots, x_{n}=y$ of points in $Z^{d}$ such that for each $k \leqq n$ the edge from $x_{k-1}$ to $x_{k}$ is open.

Once the definition of $x \rightarrow y$ is made it becomes natural to make two more definitions

$$
C_{0} \quad \text { (the cluster containing } 0 \text { ) is }\{x: 0 \rightarrow x\},
$$

* Alfred P. Sloan Fellow. This work partially supported by NSF grant MCS 80-02732. Address after July 15, 1985: Dept. of Mathematics, Cornell University, Ithaca, NY 14853, USA 
i.e. the set of all points which can be reached from $x$

$$
\Omega_{\infty}=\left\{\left|C_{0}\right|=\infty\right\}=\text { "percolation occurs" }
$$

and ask a number of questions, but we will suppress these urges until we finish describing the models. The next two are simple modifications of the first.

2. Oriented Percolation in $Z^{d}$. In this variation of 1 , if $e_{i}$ is the $i$ th unit vector then each bond is oriented $x \rightarrow x+e_{i}$ and we make the obvious change in the definition of $x \rightarrow y$ : we are only allowed to go in the direction of the orientation (and only along open bonds).

3. The Discrete Time Contact Process. In this variation of 2 we view the last coordinate as time and write $Z^{d}=Z^{n} \times Z$. Each $(x, t) \in Z^{n} \times Z$ is connected by oriented arcs to each $(x+y, t+1)$ with $|y| \leqq 1$ and the arcs are each independently open with probability $p$. In this model we interpret $(x, t) \in C_{0}$ as saying that there is a particle at $x$ at time $t$ so we view the presence of a bond from $(x, t)$ to $(x, t+1)$ as saying that the particle survived, and a bond from $(x, t)$ to $(x+y, t+1)$ as saying that the particle gave birth to a new particle at $x$ $+y$ (subject to the restriction of there being only one particle per site). For more about this interpretation see Griffeath (1979) or Durrett (1984).

Last but not least we have the example which will be used to give bounds on the others.

4. Percolation on the Binary Tree ( $a$ Galton-Watson Process). Let $S$ be the set of finite sequence of 0 's and 1's (including the sequence which has length zero) and connect each $x \in S$ by an oriented arc to the two strings $x 0$ and $x 1$ which are obtained by adding a 0 or 1 at the end. If each edge is independently open with probability $p$ and we let $Z_{n}$ be the number of $x \in C_{0}$ with length $n$ then $Z_{n}$ is a Galton Watson process with offspring distribution given by $p_{0}=(1-p)^{2}, p_{1}$ $=2 p\left(1-p\right.$ ), and $p_{2}=p^{2}$ (so the mean $=2 p$ ).

The four models described above are always included when we say below that our results are valid for "any" model and it will not be hard to see from the proofs that the results can be extended to other related which have been considered. In particular (a) we can consider percolation problems in which sites as well as bonds are open, (b) we can replace $Z^{d}$ by a "periodic graph" (for a precise definition see Kesten (1982) Chap.2), (c) in example 3 we can make different types of edges open with dirrerent probabilities and construct a large family of discrete time growth models, (d) we can take limits of systems in (c) to construct continuous time processes (for more about this see Griffeath (1981)), and (e) in example 4 we can consider more general branching distributions (e.g. the results of Erdös and Renyi graphs correspond to those for Galton-Watson processes with Poisson offspring distributions, see Spencer (1973)).

However, proving our results in a way that accomodates all these examples is unthinkable. It would strain our notation and the readers willingness to believe our proofs to the breaking point, so we will only prove the results for the four examples. In Sect. 2 we will state the results we have obtained. In Sect. 3 we will prove the results for percolation on the binary tree, and in Sects. 4 and 5 we will prove the results for the other three models. 


\section{Definitions of Critical Exponents, Description of Main Results}

Having described the percolation processes that we will consider, our next step is to introduce the "critical exponents" we referred to above. In doing this we will use the notation which is now standard in the physics literature and we would like to encourage others to do so because it facilitates comparing results for percolation with their analogues for the Ising model.

Perhaps the most basic quantities associated with a percolation process are $P\left(\Omega_{\infty}\right)=$ the probability of percolation and $p_{c}=\inf \left\{p: P\left(\Omega_{\infty}\right)>0\right\}$, so we will start by defining the corresponding exponent: $\beta$. Intuitively $\beta$ measures the rate (i.e. power of $\left.p-p_{c}\right)$ at which $P\left(\Omega_{\infty}\right) \downarrow 0$ when $p \downarrow p_{c}$. In most examples we expect to find

where $\sim$ means

$$
P\left(\Omega_{\infty}\right) \sim C\left(p-p_{c}\right)^{\beta}
$$

$$
\lim _{p \downarrow p_{c}} \frac{P\left(\Omega_{\infty}\right)}{C\left(p-p_{c}\right)^{\beta}}=1
$$

but this notion is too strong for some examples (and too hard to prove) so we will replace $\sim$ by the weaker notion

where $\approx$ means

$$
P\left(\Omega_{\infty}\right) \approx\left(p-p_{c}\right)^{\beta}
$$

$$
\lim _{p \downarrow p_{c}} \frac{\log P\left(\Omega_{\infty}\right)}{\beta \log \left(p-p_{c}\right)}=1 .
$$

The second definition allows logarithmic factors (or slowly varying functions) or much worse behavior, but as we will see below, in most cases we do not know that the limit used to define $\beta$ exists. To be able to talk about $\beta$ without assuming its existence we will extend the definition above and say

$$
\begin{aligned}
& \beta \leqq c \quad \text { if } \limsup _{p \downarrow p_{c}} \frac{\log P\left(\Omega_{\infty}\right)}{\log \left(p-p_{c}\right)} \leqq c \\
& \beta \geqq c \quad \text { if } \liminf _{p \downarrow p_{c}} \frac{\log P\left(\Omega_{\infty}\right)}{\log \left(p-p_{c}\right)} \geqq c .
\end{aligned}
$$

For percolation on the tree it is easy to compute $\beta$. In this case the theory of branching processes tells us that $1-P\left(\Omega_{\infty}\right)$ is the smallest solution of $((1-p)$ $+p x)^{2}=x$ with $x \in[0,1]$ (see Athreya and Ney (1972), Chap. 1), so a little algebra gives

$$
P\left(\Omega_{\infty}\right)= \begin{cases}2 p^{-1}-p^{-2} & p \geqq \frac{1}{2} \\ 0 & p \leqq \frac{1}{2} .\end{cases}
$$

Differentiating the formula for $p \geqq \frac{1}{2}$ gives $-2\left(p^{-2}-p^{-3}\right)$ which is 8 at $p=\frac{1}{2}$ so we have

$$
P\left(\Omega_{\infty}\right) \sim 8\left(p-p_{c}\right) \quad \text { as } p \downarrow p_{c}
$$

and the critical exponent $\beta=1$. 
Differentiating $-2\left(p^{-2}-p^{-3}\right)$ again explains why $\beta \leqq 1$ in this case. The derivative is $4 p^{-3}-6 p^{-4}=(4 p-6) p^{-4}$ which is negative for $p<\frac{3}{2}$ so $\theta(p) \equiv P\left(\Omega_{\infty}\right)$ is concave on $\left[p_{c}, 1\right]$ and hence we must have

$$
\liminf _{p \downarrow p_{c}} \frac{P\left(\Omega_{\infty}\right)}{p-p_{c}}>0 .
$$

We believe that the last conclusion and probably the one before it are true in general, but at this point in time the only argument we can give for this viewpoint is that it would be in keeping with the pattern established by the results for $\gamma, \gamma^{\prime}$, and $\Delta$ below. The results which have been shown are so weak that we don't know that $\beta \leqq 1$ in ANY example (except for percolation on trees).

For bond and site percolation on $Z^{2}$ (and some other models) Kesten (1981) has shown that there are constants $C_{i}, \alpha_{i} \in(0, \infty)$ so that

$$
C_{3}\left(p-p_{c}\right)^{\alpha_{3}} \leqq P\left(\Omega_{\infty}\right) \leqq C_{4}\left(p-p_{c}\right)^{\alpha_{4}} .
$$

The proof of this fact and in particular the proof of the lower bound, requires a lot of ingenuity, but the $\alpha_{i}$ which are obtain are not very good. Tracing back through the proof of the upper bound gives (in Kesten's notation)

$$
\alpha_{4}=\frac{\beta_{0}}{2}=\frac{-\log \left(1-\gamma_{0}\right)}{2 \log 3}
$$

where $\gamma_{0}$ is the probability of a special event which is trivially $\leqq\left(\frac{1}{2}\right)^{4}$ (for bond percolation) and for which a typical lower bound is $2^{-12}(0.2929)^{64}$ (see Smythe and Wierman (1978), p. 41). The lower bound is harder to compute $\left(\alpha_{3}=\right.$ $\left.-2\left(\alpha_{12}-1\right) C_{19}=\ldots\right)$ and is probably just as bad, but in any case it is clear from the proof that it is much larger than 1.

Our knowledge of $P\left(\Omega_{\infty}\right)$ for oriented percolation in $Z^{2}$ is better and worse. We have shown (see Durrett (1984), Sect. 5) that

$$
P\left(\Omega_{\infty}\right) \geqq 4\left(p-p_{c}\right)^{2}
$$

so the lower bound is much better (although still not $\geqq C\left(p-p_{c}\right)$ ) but on the other hand we do not know whether or not

$$
P\left(\Omega_{\infty}\right) \downarrow 0 \quad \text { as } p \downarrow p_{c} \text {. }
$$

This question is also an open problem for bond and site percolation in $Z^{d}$ when $d \geqq 3$.

After the percolation probability the next most important quantity associated with percolation is the mean cluster size $E\left|C_{0}\right|$. This quantity has its own critical value

$$
p_{T}=\inf \left\{p: E\left|C_{0}\right|=\infty\right\}
$$

which is possibly different from $p_{c}$ (but usually isn't and trivially satisfies $p_{T} \leqq p_{c}$ ) so we define 


$$
\gamma=-\lim _{p \uparrow p_{T}} \frac{\log E\left|C_{0}\right|}{\log \left(p_{T}-p\right)}
$$

(with the conventions discussed above in force when the limit does not exist).

For percolation on the tree

$$
E\left|C_{0}\right|=\sum_{n=0}^{\infty}(2 p)^{n}=(1-2 p)^{-1}
$$

so $p_{T}=\frac{1}{2}\left(=p_{c}\right)$ and as $p \uparrow p_{T}$

$$
E\left|C_{0}\right|=\left(2\left(p_{T}-p\right)\right)^{-1}
$$

and the critical exponent $\gamma=1$. Recently Aizenman and Newman (1984) have shown that this behavior gives a lower bound on the singularity for bond percolation. We will give a version of their proof below based on van den Berg's inequality which makes it clear that the result is valid for "any" model. For another version of this proof see van den Berg and Kesten (198).

To state the lower bound and describe its ramifications, we need some notation. Let $\chi=E\left|C_{0}\right|$. The key to proving $\gamma \geqq 1$ is to show

$$
\frac{d \chi}{d p} \leqq \kappa \chi^{2}
$$

where $\kappa=$ the coordination number $=$ the number of points which can be reached from 0 in one step. From this it follows immediately that

so we have

$$
0 \geqq \frac{d \chi^{-1}}{d p} \geqq-\kappa
$$

$$
\chi \geqq\left(\kappa\left(p_{T}-p\right)\right)^{-1}
$$

(which becomes an equality for percolation on the tree).

The last result sharpens one side of an inequality proved by Kesten (1981) for two dimensional percolation processes

$$
C_{5}\left(p_{T}-p\right)^{-\alpha_{5}} \leqq E\left|C_{0}\right| \leqq C_{6}\left(p_{T}-p\right)^{-\alpha_{6}}
$$

(again $\alpha_{5}$ is rather small and is trivially less than 1, see (3.46) in Kesten's paper). The inequality (1) which led to (2) is also useful by itself for it allows us to conclude that $\chi^{-1}$ is Lipshitz continuous and hence

$$
\text { As } \quad p \uparrow p_{T}, \chi \uparrow \infty \text {. }
$$

This result (which was first proved for percolation on periodic graphs by Kesten (1981), see Corollary 2 in his paper) is in sharp contrast to the state of affairs for the percolation probability described above. There we do not know if we have in general

$$
P\left(\Omega_{\infty}\right) \downarrow 0 \quad \text { as } p \downarrow p_{c} .
$$
let

When $p>p_{c}, E\left|C_{0}\right|=\infty$ for the trivial reason that $P\left(\left|C_{0}\right|=\infty\right)>0$, so if we

$$
S(p)=E\left(\left|C_{0}\right| ;\left|C_{0}\right|<\infty\right)
$$


we have a hope of getting something which is not $\equiv \infty$ for $p>p_{c}$ and in fact, for percolation on the tree, it is not hard to show (see Sect. 3) that if $p>p_{c}$.

$$
S(p)=\left(1-P\left(\Omega_{\infty}\right)\right) S(1-p)<\infty .
$$

So it follows that as $p \downarrow p c$

$$
S(p) / S(1-p) \rightarrow 1
$$

i.e. in this case $S(p)$ has the same singularity as $p \downarrow p_{c}$ or $p \uparrow p_{c}$. The hope that this might be true in general and the skepticism that it might not lead to the following definition

$$
\gamma^{\prime}=-\lim _{p \downarrow p_{c}} \frac{\log S(p)}{\log \left(p-p_{c}\right)}
$$

(with the usual conventions in force when the limit does not exist).

For percolation on the tree we have $\gamma^{\prime}=1$ so by analogy with (2) we might hope to show $\gamma^{\prime} \geqq 1$. The next result proves more and less than this

If $P\left(\Omega_{\infty}\right) \downarrow 0$ as $p \downarrow p_{c}$ then for all $\varepsilon>0$

$$
\int_{p_{c}}^{p_{c}+\varepsilon} R(p) d p=\infty
$$

where $R(p) \leqq \kappa S(p)$ is size of the "external boundary" of $C_{0}$ (see Sect. 5 for a precise definition).

Again there are results of Kesten (1981) concerning two dimensional models, which continuing the pattern established above may be written as

$$
C_{7}\left(p-p_{c}\right)^{-\alpha_{7}} \leqq E\left(\left|C_{0}\right| ;\left|C_{0}\right|<\infty\right) \leqq C_{8}\left(p-p_{c}\right)^{-\alpha_{8}} .
$$

This time however the form of our result does not allow us to improve the lower bound. In view of the conjectured equality of $\gamma$ and $\gamma^{\prime}$ it is interesting to note that Kesten's argument leads to $\alpha_{5}=\alpha_{7}, \alpha_{6}=\alpha_{8}$.

Having considered the divergence of $E\left|C_{0}\right|$ as $p \uparrow p_{T}$ and $p \downarrow p_{c}$ it becomes natural to ask about the divergence of the higher moments. The first and most basic question is: Do all the moments $E\left|C_{0}\right|^{k} k=1,2, \ldots$ blow up at the same point? The next result shows that this is correct and provides a bound on $P\left(\left|C_{0}\right|>n\right)$.

If $p<p_{T}$ and $\varepsilon=\frac{\ln 2}{2} \chi^{-1}$ then

$$
P(0 \rightarrow x) \leqq 2 \exp (-\varepsilon|x|)
$$

With the simultaneous divergence of the $E\left|C_{0}\right|^{k}$ established we can define the corresponding critical exponent(s). I have put the $s$ in parentheses because we will define a sequence of "gap exponents" by

$$
\frac{E\left|C_{0}\right|^{k}}{E\left|C_{0}\right|^{k-1}} \approx\left(p_{T}-p\right)^{-\Delta_{k}} \quad \text { as } p \uparrow p_{T}
$$

but it is widely believed (by many physicists at least) that all the $\Delta_{k}$ are equal. 
To try to convince you that this is true we let

$$
\begin{aligned}
& a_{n}(p)=P\left(\left|C_{0}\right|=n\right) \\
& b_{n}(p)=n a_{n}(p) / S(p)
\end{aligned}
$$

and let $Y_{p}$ be a random variable with

$$
P\left(Y_{p}=n\right)=b_{n}(p) .
$$

It is easy to explain our interest in $Y_{p}$. If $k \geqq 2$

$$
\begin{aligned}
E Y_{p}^{k-1} & =\sum_{n} n^{k} a_{n}(p) / S(p) \\
& =E\left|C_{0}\right|^{k} / S(p) .
\end{aligned}
$$

From this observation it is only a small leap to the conclusion that $\Delta_{k} \equiv \Delta$, for if we have

$$
Y_{p} /\left(p_{T}-p\right)^{-4} \Rightarrow \text { a non-zero limit }
$$

and everything goes well then

$$
E\left|C_{0}\right|^{k} \sim S(p) E Y_{p}^{k-1} \approx\left(p_{T}-p\right)^{-\gamma-(k-1) \Delta} .
$$

For percolation on the tree $a_{n}(p)$ can be found explicitly and then it is a simple matter to conclude that as $p \uparrow p_{T}$

and that

$$
P\left(Y_{p} \leqq y 4^{-1}\left(p_{T}-p\right)^{-2}\right) \rightarrow \int_{0}^{y} C x^{-\frac{1}{2}} e^{-x} d x
$$

$$
E\left|C_{0}\right|^{k} \sim A_{k}(p-p)^{-1-(k-1) 2}
$$

where $A_{k}$ is a constant which depends upon $k$.

The proof of the last result relies very heavily on the fact that we have an explicit formula for $a_{n}(p)$ so we are far from proving anything like this in general. However in keeping with the theme of the paper the behavior on the tree provides bounds on one side for what happens in general. Aizenman and Newman (1984) have also shown that

$$
\limsup _{p \uparrow p T} E\left(\frac{Y_{p}}{S(p)^{2}}\right)^{j} \leqq m_{j}
$$

where $m_{j}$ is the $j$ th moment of the chi-square distribution.

(7) is a simple corollary of some inequalities called "tree graph inequalities," which in turn are (as we will show in Sect. 4) are simple consequences of van den Berg's lemma. This result is yet another indication that quantities for the tree should bound those quantities in general. Looking back at (6) we see that the norming is $4^{-1}\left(p_{T}-p\right)^{-2}=S(p)^{2}$ and that the limit is indeed a chi square, but it is $\frac{1}{2}$ the square of a standard normal.

As a corollary of (7) we see that for Kesten's two dimensional models when $p<p_{T}$

$$
\left(C_{5}\left(p_{T}-p\right)^{-\alpha_{5}}\right)^{k} \leqq E\left|C_{0}\right|^{k} \leqq\left(C_{6}\left(p_{T}-p\right)^{-\alpha_{6}}\right)^{2 k+1},
$$


the first inequality being due to Jensen. Extending the reasoning used to obtain these inequalities it is easy to show that $\Delta_{k}$ is an increasing function of $k \geqq 2$ and satisfies

$$
\gamma \leqq \Delta_{k} \leqq 2 \gamma
$$

(see Sect. 5 for details).

Combining the last result with (2) gives $A_{k} \geqq 1$ but if you trust (as we do) the philosophy that the exponents for the tree should provide bounds on one side for what happens in general you realize that something stronger must be true

$$
\Delta_{2} \geqq 2
$$

and it is. This fact was discovered by one of my students (B. Nguyen) and is proved in our joint paper (see references).

\section{Percolation on the Binary Tree}

In this section we will prove the statements we have made concerning percolation on the tree. The facts concerning $\beta$ and $\gamma$ were proved in Sect. 2, so we will begin by showing that $\gamma^{\prime}=1$. To do this we observe that if $Z_{n}$ is the number of wet sites on level $n$ (see Sect. 1 for a precise definition) then $Z_{n}$ is a Markov chain with transition probability

$$
p_{i j}=\left(\begin{array}{c}
2 i \\
j
\end{array}\right) p^{j}(1-p)^{2 i-j}
$$

so a simple application of the Markov property shows that if we let $\bar{Z}_{n}$ be $Z_{n}$ conditioned on $\Omega_{\infty}^{c}$ then $\overline{\mathcal{Z}}_{n}$ is a Markov chain with transition probability.

where $Q(p)=P\left(\Omega_{\infty}^{c}\right)$.

$$
\bar{p}_{i j}=Q(p)^{-i} p_{i j} Q(p)^{j}
$$

Unscrambling the definition gives

$$
Q(p)=1-\left(\frac{2}{p}-\frac{1}{p^{2}}\right)=\left(\frac{p-1}{p}\right)^{2}
$$

and when we plug this in a minor miracle occurs

$$
\begin{aligned}
\vec{p}_{i j} & =\left(\frac{p}{1-p}\right)^{2 i}\left(\begin{array}{c}
2 i \\
j
\end{array}\right) p^{j}(1-p)^{2 i-j}\left(\frac{1-p}{p}\right)^{2 j} \\
& =\left(\begin{array}{c}
2 i \\
j
\end{array}\right) p^{2 i-j}(1-p)^{j}
\end{aligned}
$$

so the conditioned chain is obtained from the original one by changing the parameter from $p>\frac{1}{2}$ to $1-p$. From the last observation we see that if $S(p)$ $=E\left(\left|C_{0}\right| ;\left|C_{0}\right|<\infty\right)$ then for $p>\frac{1}{2}$

$$
S(p)=Q(p) S(1-p)
$$


which proves the desired result since $Q(p) \rightarrow 1$ as $p \downarrow p_{c}$ and $S(1-p) \sim C\left(p-p_{c}\right)^{-1}$. From the argument above it follows immediately that if we let

$$
S_{k}(p)=E\left(\left|C_{0}\right|^{k} ;\left|C_{0}\right|<\infty\right)
$$

then for $p>p_{c}, S_{k}(p)=Q(p) S_{k}(1-p)$ so the divergence of $S_{k}(p)$ as $p \uparrow p_{c}$ or $p \downarrow p_{c}$ is exactly the same right down to the value of the constant.

Having dispensed with $\gamma^{\prime}$ our next task is to show that $\Delta=2$. The first step in doing this is to compute $a_{n}(p)=P\left(\left|C_{0}\right|=n\right)$. To do this we use an observation due to $M$. Dwass (1969).

Let $X_{1}, X_{2}, \ldots$ be i.i.d. with

$$
P\left(X_{i}=k\right)=\left(\begin{array}{l}
2 \\
k
\end{array}\right) p^{k}(1-p)^{2-k} \quad k=0,1,2 .
$$

Let $S_{k}=1+\sum_{j=1}^{k}\left(X_{j}-1\right)$.

Let $\tau=\inf \left\{k: S_{k}=0\right\}$.

Then we have

$$
a_{n}(p)=P(\tau=n) .
$$

The original proof of this was not very transparent (see e.g. Jagers (1975), p. 40-41) but if you see the trick (due to Bondesson (1979)) the result is easier to prove than it is to state. Since the proof is nice and short and has only appeared in Swedish we give it here.

Proof. Consider a modification of the Galton Watson process in which only one individual is chosen to reproduce at each time. If we start with one particle at time 0 then $S_{k}$ gives the number of particles alive at time $k$ in the modified system. Clearly the total number of births is the same in both processes but when only one particle dies at a time we have time of extinction $=$ the number of births +1 (for the particle at time 0 ).

With (1) established it is easy to compute $a_{n}(p)$ and find the limit of the rescaled cluster distributation. If we have a sample path with $S_{0}=1$ and $S_{n}=0$ then exactly one cyclic permutation of the increments has the property that $S_{m}>0$ for $m<n$ so

$$
P(\tau=n)=\frac{1}{n} P\left(S_{n}=0\right)
$$

(this is also a consequence of the ballot theorem, see Feller (1968), p. 73) and hence

$$
\begin{aligned}
a_{n}(p) & =\frac{1}{n}\left(\begin{array}{c}
2 n \\
n-1
\end{array}\right) p^{n-1}(1-p)^{n+1} \\
b_{n}(p) & =\left(\begin{array}{c}
2 n \\
n-1
\end{array}\right) p^{n-1}(1-p)^{n+1} /(1-2 p)^{-1} \\
& =(1-2 p)\left(\begin{array}{c}
2 n \\
n-1
\end{array}\right)\left(\frac{1}{2}\right)^{2 n}(4 p(1-p))^{n} \frac{(1-p)}{p} .
\end{aligned}
$$


Now Stirling's formula (or our knowledge of simple random walk) shows

$$
\left(\begin{array}{c}
2 n \\
n-1
\end{array}\right)\left(\frac{1}{2}\right)^{2 n} \sim C n^{-\frac{1}{2}}
$$

and if we let $\varepsilon=\frac{1}{2}-p$ then

$$
4 p(1-p)=4\left(\frac{1}{2}-\varepsilon\right)\left(\frac{1}{2}+\varepsilon\right)=1-4 \varepsilon^{2} .
$$

Combining the last two observations with the formula for $b_{n}(p)$ suggest taking $n \approx \varepsilon^{-2}$. Doing this leads to

$$
\begin{aligned}
P\left(Y_{p}=y \varepsilon^{-2}\right) & \sim(2 \varepsilon)\left(C y^{-\frac{1}{2}} \varepsilon\right)\left(1-4 \varepsilon^{2}\right) y \varepsilon^{-2} / 4 \\
& \sim \varepsilon^{2} 2 C y^{\frac{1}{2}} e^{-4 y}
\end{aligned}
$$

from which it follows easily that for $0<a<b<\infty$

$$
P\left(a \varepsilon^{-2} \leqq y \leqq b \varepsilon^{-2}\right) \rightarrow \int_{a}^{b} 2 C y^{-\frac{1}{2}} e^{-4 y} d y,
$$

which after a change of variables is (6) of Sect. 2. We leave it to the reader to check that we can conclude from this that

$$
E\left|C_{0}\right|^{k} \sim A_{k}\left(p_{T}-p\right)^{-1-(k-1) \Delta} .
$$

\section{Some Useful Inequalities}

In this section we will describe some inequalities which have been proved recently and which are the key to obtaining the bounds given in the next section. The most important of these is what we call van den Berg's inequality. For simplicity we will only state a special case of his result

If $x_{i}, y_{i} \in Z^{d}$ for $i=1, \ldots, n$ then

$$
P\left(x_{i} \rightarrow y_{i} \text { pairwise disjoint paths }\right) \leqq \prod_{i=1}^{n} P\left(x_{i} \rightarrow y_{i}\right) .
$$

Proof. See van den Berg and Kesten (198 ). The result is stated for increasing functions of independent random variables so it is clear that it is valid for "any" model.

You will see in a minute that this result is a useful complement to the statement we get from Harris' FKG inequality (see Kesten (1982), Sect. 4.1).

$$
P\left(x_{i} \rightarrow y_{i} \text { for } i=1, \ldots, n\right) \geqq \prod_{i=1}^{n} P\left(x_{i} \rightarrow y_{i}\right) .
$$

From (1) we get a number of useful corollaries. To state the first of these we need a definition. We say that $A \subset Z^{d}$ separates $x$ and $y$ if $x, y \notin A$ but any path connecting $x$ and $y$ contains an element of $A$. 
Simon's Inequality. If $A$ separates $x$ and $y$ then

$$
P(x \rightarrow y) \leqq \sum_{z \in A} P(x \rightarrow z) P(z \rightarrow y) .
$$

Remark. This inequality is named after its analogue for the Ising model given in Simon (1980). The percolation analogue was discovered independently by a number of people and has consequently not yet been published so we will prove it.

Proof. If $x \rightarrow y$ then there is a nonself intersecting path from $x$ to $y$. If $z$ is the first point in $A$ on this path then there are disjoint paths from $x$ to $z$ and from $z$ to $y$ so applying (1) and summing over $z$ gives (3).

Remark (Lieb's improvement). For some purposes it is useful to note that the argument above proves more. If $B$ is the component of $A^{c}$ which contains $x$ and $P_{B}(x \rightarrow z)$ is the probability $x$ is connected to $z$ within $B$ then

$$
P(x \rightarrow y) \leqq \sum_{z \in A} P_{B}(x \rightarrow z) P(z \rightarrow y) .
$$

From (3) we immediately get

If $p<p_{T}$ and $\varepsilon=\frac{\ln 2}{2} \chi^{-1}$ then

$$
P(0 \rightarrow x) \leqq 2 \exp (-\varepsilon|x|) .
$$

Proof. The proof is based on the following observation (Theorem 1.3 in Simon (1980)).

Let $f$ be a nonnegative bounded function on $Z^{d}$ and suppose that for some subset $B \subset\{x:|x| \leqq R\}$ and all $y$ with $|y|>R$ we have

$$
f(y) \leqq \sum_{x \in B} a(x) f(y-x)
$$

where $a(x) \geqq 0$ and $\sum_{x \in B} a(x)=A_{0}<1$ then

$$
|f(x)| \leqq\|f\|_{\infty} \exp \left(-m_{0} R[|x| / R]\right)
$$

where $m_{0}=-R^{-1} \ln A_{0}$.

To get (4) from (5) let $B_{n}=\{x:|x|=n\}$ and let

$$
N=\inf \left\{n: \sum_{x \in B_{n}} P(0 \rightarrow x) \leqq \frac{1}{2}\right\} .
$$

Clearly $\chi \geqq N / 2$. Applying (5) with $B=B_{N}$ then gives

$$
P(0 \rightarrow x) \leqq \exp \left(-m_{0}[|x| / N]\right)
$$

where $m_{0}=N^{-1} \log 2$ so we have

$$
\begin{aligned}
P(0 \rightarrow x) & \leqq 2 \exp (-\log 2(|x| / N)) \\
& \leqq 2 \exp \left(-\frac{\log 2}{2 \chi}|x|\right) .
\end{aligned}
$$


Extending the reasoning above to three points gives an inequality due to Aizenman and Newman (1981)

$$
P\left(x, y \in C_{0}\right) \leqq \sum_{z} P(0 \rightarrow z) P(x \rightarrow z) P(y \rightarrow z) .
$$

Proof. By van den Berg's lemma it suffices to show that if $x, y \in C_{0}$ then there is a point $z \in C_{0}$ which is connected to $0, x$, and $y$ by disjoint paths. To find $z$ systematically remove bonds from $C_{0}$ making sure that the deleted set is always connected. The first time $x$ can no longer be reached from $y$ you have found $z$.

Extending the reasoning above to $n$ points we get a sequence of inequalities due to Aizenman and Newman, which are called the tree graph inequalities. The first of these is

$$
\begin{aligned}
P\left(x_{1}, x_{2}, x_{3} \in C_{0}\right) \leqq & \sum_{y, z} P\left(\begin{array}{c}
0-y-1 \\
1 \\
2-z-3
\end{array}\right) \\
& +P\left(\begin{array}{c}
0-y-2 \\
\mid \\
1-z-3
\end{array}\right) \\
& +P\left(\begin{array}{c}
0-y-3 \\
\mid \\
1-z-4
\end{array}\right)
\end{aligned}
$$

where on the right hand side $0,1,2,3$ are shorthand for $0, x_{1}, x_{2}, x_{3}$ and the lines stand for disjoint paths connecting the indicated sites.

From this example it should be clear what the general pattern is so we will only state the general result in words:

$P\left(x_{1}, \ldots, x_{n} \in C_{0}\right) \leqq$ the sum of probabilities of all the tree graphs which end in $0, x_{1}, \ldots, x_{n}=$ the number of ways of connecting these points "two at a time".

We have given only a vague description of the result because we think it is clearer than a precise one. The reader who is bothered by the ambiguity should be comforted by the fact that we will use this result only once below and at that time we will give some more details.

\section{Bounds for Percolation Processes on $Z^{d}$}

In this section we will prove the statements we made in Sect. 2 concerning the critical exponents for Examples 1-3. For concreteness and simplicity we will prove our results first for bond percolation and then show that the same proof works for the two oriented examples with the only change being that some of the steps are simpler.

We will begin by proving that $\gamma \geqq 1$. By remarks in Sect. 2 , it is enough to show that

$$
\frac{d \chi}{d p} \leqq \kappa \chi^{2}
$$


where $\kappa=$ the coordination number of the lattice. The starting point for the proof is the observation that

$$
\chi=E\left|C_{0}\right|=\sum_{x} P(0 \rightarrow x) .
$$

Differentiating with respect to $p$ (and postponing for a moment the justification of this step) gives

$$
\frac{d \chi}{d p}=\sum_{b} \sum_{x} \frac{\partial P(0 \rightarrow x)}{\partial p_{b}}
$$

where the first sum is over all the bonds and $p_{b}$ indicates the probability assigned to $b$ in a general nonhomogeneous percolation model.

To evaluate the derivative in the sum above we use Russo's formula (see Kesten (1982), Sect. 4.2) to conclude that

$$
\frac{\partial P(0 \rightarrow x)}{\partial p_{b}}=P(b \text { is "pivotal" for }\{0 \rightarrow x\})
$$

where the word in quotation marks means that changing the state of $b$ changes the occurence of $\{0 \rightarrow x\}$. It is easy to see that in order for $b$ to be pivotal for $\{0 \rightarrow x\}, 0$ must be connected to one end of $b$ (call it $y$ ), $x$ must be connected to the other (call it $z$ ), and there must be no path from $0 \rightarrow x$ which does not contain $b$. The last fact implies that any paths from $0 \rightarrow y$ and $z \rightarrow x$ must be disjoint so if we write $b=\left(b_{1}, b_{2}\right)$ then van den Berg's lemma implies that

$$
\frac{\partial P(0 \rightarrow x)}{\partial p_{b}} \leqq P\left(0 \rightarrow b_{1}\right) P\left(b_{2} \rightarrow x\right)+P\left(0 \rightarrow b_{2}\right) P\left(b_{1} \rightarrow x\right) .
$$

If we use (2) and sum the last inequality first over $x$ and then $b$ we get

$$
\frac{d \chi}{d p} \leqq \chi\left(\sum_{b} P\left(0 \rightarrow b_{1}\right)+P\left(0 \rightarrow b_{2}\right)\right)=\kappa \chi^{2}
$$

so the only detail which remains is to justify (2). To do this we begin by observing that the result is true if we are considering percolation in $\{-n, \ldots, n\}^{\mathrm{d}}=\Lambda_{n}$, and then we pass to the limit using the following

If $f_{i}$ is a sequence of differentiable functions which has the property that for some $\delta>0 \sum f_{i}$ and $\sum f_{i}^{\prime}$ converge uniformly in $[p-\delta, p+\delta]$ then $\sum f_{i}$ is differentiable at $p$ and

$$
\left(\sum f_{i}(p)\right)^{\prime}=\sum f_{i}^{\prime}(p) .
$$

For a proof of this (see Durrett (1984), Sect. 15). To get (2) from (4) let $f_{i}(p)$ $=P\left(0 \rightarrow x\right.$ by a path which lies in $\Lambda_{i}$ but not in $\left.\Lambda_{i-1}\right)$ and use (3) and the bound

$$
P(0 \rightarrow x) \leqq 2 \exp (-\varepsilon|x|)
$$

proved in the last section to check uniform convergence. The details are somewhat tedious and left to the reader. 
Remark. From the proof of (1) given above the reader can see that the same proof works for Examples 2 and 3 and in fact the argument simplifies for oriented percolation since we know which ends of $b, 0$ and $x$ will be connected to and if $b=(y, z)$ there is no need to use van den Berg's inequality since we have

$$
P(0 \rightarrow y, z \rightarrow x)=P(0 \rightarrow y) P(z \rightarrow x) .
$$

Having established the behavior of $E\left|C_{0}\right|$ as $p \uparrow p_{T}$ we now turn our attention to what happens when $p \downarrow p_{c}$. Following Sokal (1981) (see p. 33) we will prove our result by looking at $M(p)=P\left(\Omega_{\infty}\right)$. (The $M$ stands for magnetization, the Ising model analogue of the percolation probability.) Differentiating with respect to $p$ (this time leaving the justification to the reader) and applying Russo's formula gives

$$
\begin{aligned}
0 & \leqq \frac{\partial M}{\partial p}=\sum_{b} P(b \text { is pivotal for } 0 \rightarrow \infty) \\
& =\sum_{(x, y)} P(0 \rightarrow x, y \rightarrow \infty \text { but if }(x, y) \text { is closed then } 0 \rightarrow \infty)
\end{aligned}
$$

where the sum is over all ordered pairs of neighboring sites.

A little thought (or a look at Kesten (1982), p. 79) shows that the event " $b$ is pivotal for $0 \rightarrow \infty$ " is independent of the state of $b$ so we can write the last expression as

$$
\frac{1}{(1-p)} \sum_{(x, y)} P\left(\begin{array}{l}
(x, y) \text { is closed, }\left|C_{0}\right|<\infty \\
x \in C_{0} \text { and } y \rightarrow \infty
\end{array}\right) .
$$

To bound the probability in the sum it is convenient to break things down according to the exact shape of $C_{0}$. Let $K(0, x ; y)$ be the set of finite clusters $A$ which (i) contain 0 and $x$, (ii) do not contain $y$ (and hence not $(x, y)$ ) and furthermore (iii) $y$ can be connected to $\infty$ in $A^{c}$. With this notation we can write the probability above as

$$
\begin{aligned}
& \sum_{A \in K(0, x ; y)} P\left(C_{0}=A, y \rightarrow \infty\right) \\
& \sum_{A \in K(0, x ; y)} P\left(C_{0}=A\right) P\left(y \rightarrow \infty \mid C_{0}=A\right) .
\end{aligned}
$$

Since $y \in A^{c}$ it is easy to see that

$$
\begin{aligned}
P\left(y \rightarrow \infty \mid C_{0}=A\right) & =P(y \rightarrow \infty \mid \text { all bonds in } \partial A \text { are closed }) \\
& \leqq P(y \rightarrow \infty)
\end{aligned}
$$

by Harris' inequality, so the last sum above

$$
\begin{aligned}
& \leqq \sum_{A \in K(0, x ; y)} P\left(C_{0}=A\right) P(y \rightarrow \infty) \\
& =M \sum_{A \in K(0, x ; y)} P\left(C_{0}=A\right) \\
& =M P\left(\begin{array}{l}
\left|C_{0}\right|<\infty, x \in C_{0}, y \notin C_{0} \text { and } y \text { can be connected } \\
\text { to } \infty \text { in } C_{0}^{c}
\end{array}\right) .
\end{aligned}
$$


Summing the last probability over $(x, y)$ and calling the result $R(p)$ gives

$$
0 \leqq \frac{\partial M}{\partial p} \leqq \frac{1}{1-p} M(p) R(p) \text {. }
$$

Near $p_{c}$ we can rewrite the last inequality as

$$
\varepsilon M^{-1} \frac{\partial M}{\partial p} \leqq R(p)
$$

where $\varepsilon>0$ so if $p_{c}<a<b$

$$
\begin{aligned}
\int_{a}^{b} R(p) d p & \geqq \varepsilon \int_{a}^{b} M^{-1} \frac{\partial M}{\partial p} d p \\
& =\varepsilon(\log M(b)-\log M(a)) .
\end{aligned}
$$

If $M(p) \rightarrow 0$ as $p \rightarrow p_{c}$ then letting $b=p_{c}+\varepsilon$ where $\varepsilon>0$ and $a \downarrow p_{c}$ shows

proving (4) of Sect. 2.

$$
\int_{p_{c}}^{b_{c}+\varepsilon} R(p) d p=\infty
$$

The last thing we have to do in this section is to prove that $\gamma \leqq A_{k} \leqq 2 \gamma$ for $k \geqq 2$. To do this we begin by proving the right hand inequality for $k=2$. To prove the result in this case we use the first tree graph inequality

$$
\begin{aligned}
E\left|C_{0}\right|^{2} & =\sum_{x, y} P\left(x, y \in C_{0}\right) \\
& \leqq \sum_{x, y, z} P(0 \rightarrow z) P(x \rightarrow z) P(y \rightarrow z) .
\end{aligned}
$$

Summing first over $x$ and $y$ and then over $z$ gives

$$
E\left|C_{0}\right|^{2} \leqq\left(E\left|C_{0}\right|\right)^{3}
$$

so if the $L H S \approx\left(p_{c}-p\right)^{-\gamma-\Delta_{2}}$ and the $R H S \approx\left(p_{c}-p\right)^{-3 \gamma}$ we must have $\Delta_{2} \leqq 2 \gamma$.

To extend this to $k \geqq 3$ we will use the tree graph inequalities. To keep from drowning in notation we will prove the result first for $k=3$ and then proceed to the general case.

$$
\begin{aligned}
& E\left|C_{0}\right|^{3}=\sum_{x_{1}, x_{2}, x_{3}} P\left(x_{1}, x_{2}, x_{3} \in C_{0}\right) \\
& \leqq \sum_{x_{1}, x_{2}, x_{3}}\left(\sum_{y, z} P\left(\begin{array}{cc}
0-y-1 \\
& \mid \\
2-z-3
\end{array}\right)+2 \text { similar terms }\right) \text {. }
\end{aligned}
$$

Summing first over $x_{1}, x_{2}, x_{3}$, then over $z$ and $y$ gives

$$
E\left|C_{0}\right|^{3} \leqq 3 \chi^{5} \text {. }
$$

Looking back at the last argument and comparing (6) and (7) should suggest that in general

$$
E\left|C_{0}\right|^{k} \leqq B_{k} \chi^{2 k-1}
$$


where $B_{k}$ is the number of terms on the right hand side of the inequality. To evaluate $B_{k}$ (and explain why the exponent $=2 k-1$ ) we observe that all the tree graphs for the $k+1$ moment can be obtained by taking the tree graphs for the $k$ th moment and systematically gluing an arc ending in $k+1$ to the middle of each existing arc, so one concludes by induction that the tree graphs for the $k$ th moment have $2 k-1$ arcs and hence

$$
B_{k+1}=(2 k-1)(2 k-3) \ldots 3 \cdot 1 .
$$

With (8) established the rest is easy, for if

$$
L H S \approx\left(p_{c}-p\right)^{-\gamma-\sum_{j=2}^{k} \Delta_{j}}
$$

and

we must have

$$
R H S \approx\left(p_{c}-p\right)^{-\gamma(2 k-1)}
$$

$$
\sum_{j=2}^{k} \Delta_{j} \leqq(2 k-2) \gamma .
$$

To improve this to the desired result we observe that the Cauchy Schwarz inequality implies that if $X \geqq 0$ and $m \geqq 1$

so

$$
\begin{aligned}
\left(E X^{m}\right)^{2} & =\left(E X^{\frac{m-1}{2}} X^{\frac{m+1}{2}}\right)^{2} \\
& \leqq E X^{m-1} E X^{m+1}
\end{aligned}
$$

$$
\frac{E X^{m+1}}{E X^{m}} \geqq \frac{E X^{m}}{E X^{m-1}}
$$

Letting $X=\left|C_{0}\right|$ shows $\Delta_{m-1} \leqq \Delta_{m}$ so $k \rightarrow \Delta_{k}$ is increasing and each $\Delta_{k} \geqq \Delta_{1}=\gamma$. Letting $k \rightarrow \infty$ in (9) now shows that for any $j$

$$
\Delta_{j} \leqq \lim _{k \rightarrow \infty} \Delta_{k} \leqq 2 \gamma .
$$

Acknowledgements. It should be clear from the number of references to Aizenman and Newman (1984) and to van den Berg and/or Kesten that this paper is largely a survey of their work. The reader is invited to consult their papers and Durrett and Nguyen (198) for more on this subject.

\section{References}

Aizenman, M., Newman, C.: Tree graph inequalities and critical behavior in percolation models. J. Statist Phys. [To appear 1984]

Athreya, K., Ney, P.: Branching Processes. Berlin Heidelberg New York: Springer 1972

Bondesson, L.: Angảende problem 3129 eller nágot om spel på enarmad bandit. Elementa. 62, 129-133 (1979)

Durrett, R., Griffeath, D.: Supercritical contact processes on Z. Ann. Probab. 11, 1-15 (1983)

Durrett, R.: An introduction to oriented percolation. Ann. Probab. 12, 999-1040 (1984)

Dwass, M.: The total progeny in a branching process and a related random walk. J. Appl. Probab. 6, 682-686 (1969) 
Feller, W.: An Introduction to Probability Theory and Its Applications, Vol. I, third edition. New York: Wiley 1968

Griffeath, D.: Additive and Cancellative Interacting Particle Systems. Lecture Notes in Math. 724. Berlin-Heidelberg-New York: Springer 1979

Griffeath, D.: The basic contact processes. Stochastic Processes Appl. 11, 151-185 (1981)

Jagers, P.: Branching Processes with Biological Applications. New York: Wiley 1975

Kesten, H.: Analyticity properties and power law estimates of functions in percolation theory. J. Statist. Phys. 25, 717-756 (1981)

Kesten, H.: Percolation Theory for Mathematicians. Boston: Birkhauser 1982

Simon, B.: Correlation inequalities and the decay of correlations in ferromagnets. Comm. Math. Phys. 77, 111-126 (1980)

Smythe, R., Wierman, J.: First passage percolation on the square lattice. Lecture Notes Mathematics 671. New York-Heidelberg-Berlin: Springer 1978

Sokal, A.: More inequalities for critical exponents. J. Stat. Phys. 25, 25-50 (1981)

Spencer, J., editor: Paul Erdös: The Art of Counting. Cambridge, Mass.: MIT Press 1973

van den Berg, J., Kesten, H.: Inequalities with application to percolation and reliability. J. Appl. Prob., to appear

Durrett, R., B. Nguyen, B.: (1985) Thermodynamic inequalities for percolation [Submitted to Comm. Math. Phys. 98, to appear]

Received October 2, 1983 